\title{
Cuantificación de las Categorías de Drosófilas y Levaduras Mediante los Métodos de Promedios Recíprocos y Análisis de Varianza

\author{
Doriz Gómez ${ }^{1}$, Jorge Condado ${ }^{2}$, Liliana Huamán ${ }^{2}$, \\ Walter Acuña ${ }^{2}$, Martha Gonzales ${ }^{2}$ \& Gregoria Ramón ${ }^{2}$
}

\begin{abstract}
Resumen: Un problema latente en tablas de contingencia bidimensional es la cuantificación de los niveles de las variables categóricas involucradas. En el presente trabajo se eligen los métodos descriptivos multivariantes no lineales, el Método del Promedio Recíproco y el Método de Análisis de Varianza para la cuantificación de los niveles de drosófilas y levaduras [4], variables categóricas de la tabla de contingencia bidimensional. Los resultados encontrados muestran que fue posible la cuantificación de las categorías drosófilas y levaduras mediante las metodologías señaladas, las mismas que producen cuantificaciones diferentes a las tradicionalmente usadas para las categorías de las filas y columnas de la tabla de contingencia.
\end{abstract}

Palabras clave: Drosófilas. Método del Promedio Recíproco. Método de Análisis de varianza.

\section{Quantification of the Categories of Drosophilas and Yeast by the Methods of Reciprocal Averages and Variance Analysis}

\begin{abstract}
A latent problem in two-dimensional contingency tables is to quantify the levels of categorical variables involved. In this paper we choose the nonlinear multivariate descriptive methods, Reciprocal averaging method and Variance Analysis method for quantifying levels of drosophilas and yeast, categorical variables of twodimensional contingency table. The results show that it was possible to quantify the categories drosophilas and yeast by the methods mentioned, the same ones that produce different quantifications of the categories traditionally used for the rows and columns of the contingency table.
\end{abstract}

Key words: Drosophila. Reciprocal averaging method. Analysis of variance method.

\section{Introducción}

Cuando el comportamiento de los datos no es normal, la obtención de las relaciones lineales no recoge la componente no lineal contenida en los datos, generando que muchas veces no se capte dicha información. En tal sentido, cuantificar las relaciones no lineales entre variables se ha convertido en un tema que ha merecido la atención de muchos investigadores.

Para evitar tal pérdida de información en los datos, aparecieron los métodos descriptivos multivariantes no lineales denominados métodos MUNDA. Autores como Greenacre, Lebart y Nishisato entre otros, propusieron metodologías tales como el Análisis de Correspondencia, el Promedio Recíproco y el Análisis de Varianza.

Para estudiar relaciones de dependencia, cuando se tiene datos cualitativos presentados en tablas de contingencia, son muchos los métodos estadísticos para explorar y confirmar estructuras

\footnotetext{
${ }^{1}$ UNMSM, Facultad de Ciencias Matemáticas, e-mail: dorisgomezt@gmail.com

${ }^{2}$ UNMSM, Facultad de Ciencias Matemáticas
} 
de asociación entre individuos de una población. Entre las metodologías confirmatorias están los modelos log lineales ya ampliamente discutidos en [2] entre otros, y los métodos de mínimos cuadrados ponderados discutidos y aplicados por [8]. Estos dos métodos se basan justamente en modelos explicativos de relaciones funcionales entre las probabilidades de las celdas de la tabla de contingencia. Como metodología exploratoria importante se tiene el análisis de correspondencia, discutida y muy aplicada en [9] y [6] entre otros. El análisis de correspondencia es extremadamente útil en el estudio de estructuras de asociación y se ha utilizado con mucha frecuencia en Francia, en el estudio de grandes muestras relacionando profesiones y causas de muerte, profesiones e ítems de consumo, profesiones y lugares de esparcimiento en las vacaciones [9]. No obstante su gran aplicación y simplicidad de interpretación geométrica, la medida de disimilaridad usada con mayor frecuencia es la $j i$ cuadrado, basada en las frecuencias observadas $(o)$ y las frecuencias esperadas $(e)$, que tiene serios inconvenientes porque es unidireccional, esto es $\sum(o-e)^{2} / e \neq \sum(e-o)^{2} / o$. Este hecho hace que la matriz usada en el estudio de las asociaciones sea semidefinida positiva, lo que implica una pérdida de dimensión que puede ser determinante para entender aspectos importantes de la tabla de contingencia en la que se resume la información para variables cualitativas. Esta desventaja del análisis de correspondencia motivó a [3] a proponer la teoría de Análisis de Dependencia (ANADEP) para tablas de contingencia bidimensional y multidimensional, usando la medida de asociación $M$, entre filas y columnas de la tabla de contingencia, dada por Khan e Ali en 1973, quienes demostraron que $M$ satisface las propiedades requeridas para un coeficiente de asociación, creando matrices de dependencia que emulan matrices de correlaciones para variables cuantitativas. [3] construyó, a partir de la tabla de contingenciaparámetros como las dependencias, codependencia y coeficientes de dependencia que emulan para el caso de datos categóricos, las varianzas, covarianzas, coeficientes de correlación, correspondientes a datos numéricos, dando origen a la teoría ANADEP. También a partir de los coeficientes de dependencia postuló la teoría del Análisis Factorial de Dependencia (ANADEPF).

Mediante el Análisis de Dependencia [3], se consiguió establecer relaciones de asociación entre las especies de moscas: Drosofila Novemaristata, D. Inca, D.Huaylasi, D. Melanogaster y las levaduras-tres cepas: Saccharomyces Cerevisiac, Candida utilis y Rhodotorula sp. [6].

El objetivo del presente estudio es usar los métodos descriptivos multivariantes no lineales, método del Promedio Recíproco y el método de Análisis de Varianza en tablas de contingencia de doble entrada, a fin de encontrar la mejor escala que permita cuantificar las categorías de drosófilas y levaduras [4].

\section{Métodos Estadísticos}

\subsection{Método del Promedio Recíproco (MPR)}

En el contexto de una tabla de contingencia bidimensional, sean:

$F=\left(f_{i j}: \quad i=1, \ldots I ; \quad j=1, \ldots, J\right)$, la matriz de frecuencias absolutas conjuntas, donde $f_{i j} \leq 0$, es el número de individuos escogidos al azar, que pertenecen a los niveles $i=1, \ldots, I \mathrm{y}$ $j=1, \ldots, J$ de las características $A$ y $B$;

$f_{i \bullet}$ y $f_{\bullet j}$ los totales por filas y por columnas,

$X_{j}$ e $Y_{i}$ los pesos asociados a las columnas y a las filas, respectivamente.

El método del Promedio Recíproco se inicia imputando pesos a los niveles de las columnas, $X_{j}, j=1, \ldots, J$, o a los niveles de las filas, $Y_{i}$, de la tabla de contingencia (según elección) y a pesar de que estos pesos son arbitrarios, la sugerencia es que se incluya al cero. 
Con los pesos, $X_{j}$, de los niveles de las columnas, se calculan los pesos o ponderaciones de las filas según:

$$
Y_{i}(\text { Filas })=\frac{\sum_{j=1}^{J} f_{i j}\left(X_{j}\right)}{f_{1} \bullet}, \quad i=1, \ldots, I
$$

Luego se obtiene los valores ajustados por la media, es decir:

$$
Y_{i}=Y_{i}-M
$$

donde la media ponderada por los pesos $Y_{i}$, es: $M=\frac{\sum_{i=1}^{I} f_{i \bullet}\left(Y_{i}\right)}{n}$

Para conseguir las ponderaciones finales de las filas, se divide $Y_{i}$ por $G_{Y}$, donde $G_{Y}=$ máximo $\left|Y_{i}\right|$, o sea,

$$
Y_{i}=\frac{Y_{i}}{G_{Y}}
$$

Usando las ponderaciones obtenidas para las filas, se re-calcula las ponderaciones para las columnas según:

$$
X_{j}(\text { Columnas })=\frac{\sum_{i=1}^{I} f_{i j}\left(Y_{i}\right)}{f_{\bullet j}}
$$

Se procede a encontrar los valores ajustados por la media, es decir,

$$
X_{j}=X_{j}-N
$$

donde $N=\frac{\sum_{j=1}^{J} f_{\bullet j}\left(Y_{j}\right)}{n}$.

Para obtener las ponderaciones finales de las columnas, se divide $X_{j}$ por $G_{X}$, donde $G_{X}=$ máximo $\left|X_{j}\right|$, o sea,

$$
X_{j}=\frac{X_{j}}{G_{X}}
$$

Se repiten los pasos aneriores hasta conseguir la convergencia para los pesoso ponderaciones asignados a los niveles de las columnas y filas respectivamente, llegando a las ponderaciones óptima y al igual que a los valores a los que convergen $G_{X}$ y $G_{Y} \cdot \rho=\sqrt{G_{X} G_{Y}}$, es la máxima correlación entre filas y columnas de la tabla de contingencia [10].

La constante multiplicativa para ajustar la unidad de $Y_{i}$ es $C_{r}$, donde

$$
C_{r}=\sqrt{\frac{n}{\sum_{i=1}^{I} f_{i \bullet} Y_{I}^{2}}}
$$


y la constante multiplicativa para ajustar la unidad de $X_{j}$, está dada por $C_{c}$, donde

$$
C_{c}=\sqrt{\frac{n}{\sum_{j=1}^{J} f_{\bullet j} X_{j}^{2}}}
$$

Las ponderaciones finales se obtienen multiplicando los pesos obtenidos en el último paso de las iteracciones multiplicado por para $Y_{i}$, y por $C_{c}$ para $X_{j}$.

Los nuevos pesos se denominan "pesos o ponderaciones normalizadas" y resultan siendo:

$$
Y_{i}=C_{r} Y_{i} \quad X_{j}=C_{c} X_{j}
$$

Las ponderaciones normalizadas multiplicadas por el valor singular son las coordenadas principales o pesos proyectados:

$$
Y_{i}=\rho Y_{i} \quad X_{j}=\rho X_{j}
$$

\subsection{Método de Análisis de varianza}

Según el método de Análisis de Varianza a un criterio de clasificación, se otorgan las ponderaciones o escalas de manera que sea máxima las diferencias entre las unidades de investigación y que las diferencias dentro de ellas sea mínima. Así por ejemplo, si lo que se tienen son opiniones de estudiantes respecto a sus profesores, las opiniones de los estudiantes respecto de un profesor (dentro) deben ser lo mas parecidas, mientras que sus opiniones respecto a los profesores (entre) deben ser diversas. Este método se basa en el Principio de Consistencia Interna de Guttman (in [10]).

Sean:

$\vec{Y}=\left[\begin{array}{c}Y_{1} \\ \ldots \\ Y_{I}\end{array}\right], \vec{X}=\left[\begin{array}{c}X_{1} \\ \ldots \\ X_{I}\end{array}\right]$ los vectores con los pesos para las filas y columnas,

$F$ la matriz de datos,

$f_{R}=\left[\begin{array}{c}f_{1 \bullet} \\ f_{2 \bullet} \\ \ldots \\ f_{I_{\bullet}}\end{array}\right], f_{C}=\left[\begin{array}{c}f_{\bullet 1} \\ f_{\bullet 2} \\ \ldots \\ f_{\bullet J}\end{array}\right]$ los vectores con totales por filas y columnas de la matriz $F$,

$D_{C}$ la matriz diagonal con los totales por columnas de la matriz $F$,

$D_{R}$ la matriz diagonal con los totales por filas de la matriz $F$.

Los términos del análisis de varianza, suma de cuadrados total $\left(S S_{T}\right)$, suma de cuadrados entre grupos o entre tratamientos $\left(S S_{B}\right)$ y la suma de cuadrados dentro de los grupos o dentro de los tratamientos $\left(S S_{W}\right)$, se expresan como:

$$
S S_{T}=\vec{X}^{\prime}\left[D_{C}-\frac{f_{c} f_{c}^{\prime}}{f_{t}}\right] \vec{X} \quad S S_{B}=\vec{X}^{\prime}\left[F^{\prime} D_{R}^{-1} F-\frac{f_{c} f_{c}^{\prime}}{f_{t}}\right] \vec{X}
$$

$S S_{W}=\vec{X}^{\prime}\left[D_{C}-F^{\prime} D_{R}^{-1} F\right] \vec{X}$ sujeto a las restricciones, $f_{C}^{\prime} \vec{X}=0, S S_{T}=f_{t}$.

Se procede a encontrar los pesos de las categorías de manera que hace máximo la suma de cuadrados entre tratamientos, $S S_{B}$, sujeto a la condición $S S_{T}=f_{t}$ y la suma de pesos igual a cero,por lo que en la solución se usan multiplicadores de Lagrange.

$$
Q\left(\vec{X}, \lambda_{1}, \lambda_{2}\right)=S S_{B}-\lambda_{1}\left(S S_{T}-f_{t}\right)-\lambda_{2}\left(\vec{X}^{\prime} f_{c} f_{C}^{\prime} \vec{X}-0\right)
$$




$$
\begin{gathered}
\partial Q\left(\vec{X}, \lambda_{1}, \lambda_{2}\right) / \partial \vec{X}=0 \quad \partial Q\left(\vec{X}, \lambda_{1}, \lambda_{2}\right) / \partial \lambda_{1}=0 \quad \partial Q\left(\vec{X}, \lambda_{1}, \lambda_{2}\right) / \partial \lambda_{2}=0 \\
{\left[F^{\prime} D_{R}^{-1} F-\lambda D_{C}\right] \vec{X}=0 \rightarrow F^{\prime} D_{R}^{-1} F \vec{X}=\lambda D_{C} \vec{X}}
\end{gathered}
$$

Esta ecuación se denomina ecuación del autovalor-generalizada, de donde debe obtenerse el autovalor $(\lambda)$ y autovector $\vec{X}$ de la matriz $D_{C}$.

Se demuestra que $\lambda=\frac{\vec{X}^{\prime} F^{\prime} D^{-1} R F \vec{X}}{\vec{X}^{\prime} D_{C} \vec{X}}=\frac{S S_{B}}{S S_{T}}=\eta^{2}$, donde $\eta^{2}$ es la razón de correlación.

Aquí usaremos indistintamente los términos: razón de correlación y autovalor indistintamente.

Se introduce una nueva notación, el vector, $\vec{W}=D_{C}^{-1 / 2} \vec{X}$, y se reescribe la razón de correlación en términos del nuevo vector:

$$
\eta^{2}=\frac{\vec{W}^{\prime} D_{C}^{-1 / 2} F^{\prime} D_{R}^{-1} F D_{C}^{-1} \vec{W}}{W^{\prime} \vec{W}}=\frac{\vec{W}^{\prime} B^{\prime} B \vec{W}}{\vec{W}^{\prime} \vec{W}}=\eta^{2} \text {, donde } B=D_{R}^{-1 / 2} F^{\prime} D_{C}^{-1 / 2} .
$$

La forma estándar de la ecuación-autovalor es:

$$
\left[B^{\prime} B-\lambda I\right] \vec{W}=0 \rightarrow \text { se obtiene } \vec{W} \text { y luego } \vec{X}=D_{C}^{-1 / 2} \vec{W}
$$

La solución trivial mencionada antes corresponde a $\lambda_{1}=1$ y $\vec{W}_{0}=\overrightarrow{1}$, respecto a la matriz $B$ o a la matriz de datos $F$. Para eliminar ésta solución trivial, se continúa con el procedimiento estándar para calcular la matriz residual, digamos, la matriz $C$.

Se calcula la matriz residual:

$$
C=B^{\prime} B-\lambda_{0} \frac{\vec{W}_{0} \vec{W}_{0}^{\prime}}{\vec{W}_{0}^{\prime \prime} \vec{W}_{0}}=B^{\prime} B=\frac{D_{C}^{1 / 2} \overrightarrow{1} \mid v e c 1^{\prime} D_{C}^{1 / 2}}{f_{t}}
$$

y la primera componente es la solución de la siguiente ecuación: $\left(C-\eta^{2} I\right) \vec{W}=0$, con su máximo autovalor, digamos $\eta_{1}^{2}$ y su autovector asociado $\vec{W}_{1}$.

Considerando el máximo autovector asociado al máximo autovalor $\eta_{1}^{2}$, esto es $\vec{W}_{1}$, obtenemos el primer vector de pesos óptimos, $\vec{X}_{1}$,

$$
\vec{X}_{1}=D_{C}^{-1 / 2} \vec{W}_{1} \quad W_{1}^{\prime} W_{1}=\vec{X}_{1}^{\prime} D_{C} X_{1}=f_{t}
$$

Los escores óptimos para el sujeto $i$ en la componente 1 están dada por: $Y_{i 1}=\frac{1}{\eta_{1}} \frac{\sum_{j=1} f_{i j} X_{j 1}}{f_{i \bullet}}$, donde el vector de escores de la primera componente está dados por $\vec{Y}_{1}=\frac{1}{\eta_{1}} D_{R}^{-1} F \vec{X}$.

De manera similar se pueden expresar $S S_{T}, S S_{B}, S S_{W}$ en términos de los pesos para las filas, esto es, $\vec{Y}$, y maximizar la razón de correlación $\frac{S S_{B}}{S S_{T}}$ sujeto a la condición que la suma de los pesos de las respuestas es cero y que la suma de cuadrados de las respuestas ponderadas es igual a $f_{t}$. El resultado es la ecuación-autovalor:

$$
\left[B^{\prime} B-\lambda I\right] \vec{V} \rightarrow \text { se obtiene } \vec{V} \text { y luego } \vec{Y}=D_{R}^{-1 / 2} \vec{V}
$$

\section{Análisis y Discusión}

Se llevaron a cabo colectactas de moscas drosófilas en las localidades de Anta (Provincia de Huaraz) y Choquechaca (Provincia de Caraz), dos áreas de grandes pisos de cactáceas; con la finalidad de estudiar la especificidad en la preferencia de los nutrientes que contienen las levaduras, fuente de proteínas de las drosófilas. Cabe señalar que Drosophila y Levaduras se incluyen entre los casos mas fascinantes de asociación a nivel mundial, prestándose a estudios de coevolución ([1] y [2]).

Se pretende cuantificar las categorías de las filas y de las columna de la tabla de contingencia que contiene los resultados de las colectas de moscas drosófilas en las localidades de Anta, Provincia de Huaraz y Choquechaca, Provincia de Caraz (Gómez, 2006), mediante las metodologías presentadas. 
Tabla N¹. Clasificación de moscas Drodrosophilas y la taxonomía de sus Alimentos. Localidades de Anta y Choquechaca. Ancash, 2003

\begin{tabular}{|c|c|c|c|c|c|}
\hline \multirow{2}{*}{ LEVADURAS } & \multicolumn{5}{|c|}{ Especies de drosophila } \\
\cline { 2 - 6 } & $\begin{array}{c}\text { Huaylasi (H) } \\
\left(X_{1}=0\right)\end{array}$ & $\begin{array}{c}\text { Inca (I) } \\
\left(X_{2}=1\right)\end{array}$ & $\begin{array}{c}\text { Melonogaster (M) } \\
\left(X_{3}=2\right)\end{array}$ & $\begin{array}{c}\text { Novemaristata (N) } \\
\left(X_{4}=3\right)\end{array}$ & Total \\
\hline $\begin{array}{c}\text { Saccharomyces } \\
\text { Cerevisiae (SC) }\left(Y_{1}\right)\end{array}$ & 17 & 51 & 162 & 63 & 293 \\
\hline $\begin{array}{c}\text { Candida utilis (CV) } \\
\left(Y_{2}\right)\end{array}$ & 25 & 41 & 57 & 67 & 190 \\
\hline $\begin{array}{c}\text { Rhodotorula sp (RO) } \\
\left(Y_{3}\right)\end{array}$ & 12 & 6 & 24 & 38 & 80 \\
\hline Total & 54 & 98 & 243 & 168 & 563 \\
\hline
\end{tabular}

\subsection{Método del Promedio Recíproco (MPR)}

Se Inicia asignando pesos arbitrarios a las categorías de las columnas, así, $X_{1}=0, X_{2}=1$, $X_{3}=2, X_{4}=4$ y obteniendo los pesos ponderados de las filas, media, pesos ponderados ajustados por la media y finalmente los pesos finales ponderados de las filas. En este primer paso, los valores son $Y_{1}(S C)=1,9249, Y_{2}(C V)=1,8737, Y_{3}(R O)=2,1$,

$M=1,9325, \quad Y_{1}=Y_{1}-M=1-0,0076, \quad Y_{2}=Y_{2}-M=0,0588, \quad Y_{3}=Y_{3}-M=0,1675$

$$
\begin{gathered}
G_{Y}=\text { máximo }\left|Y_{i}\right|=0,167, \quad Y_{1}=\frac{Y_{1}}{G_{Y}}=-00454, \\
Y_{2}=\frac{Y_{2}}{G_{Y}}=\frac{-0,0588}{0,1675}=-0,3510, \quad Y_{3}=\frac{Y_{3}}{G_{Y}}=1,0 .
\end{gathered}
$$

Usando los nuevos valores como ponderaciones de las filas, se calcula los nuevos promedios para las columnas, resultando:

$$
\begin{gathered}
X_{1}(H)=0,0454, \quad X_{2}(I)=-0,1092, \quad X_{3}(M)=-0,0138, \quad X_{4}(N)=0,0692 \\
N=\frac{54(0,0454)+98 X_{2}+243 X_{3}+168 X_{4}}{29}=0,000039 \\
X_{1}=X_{1}-N=0,0454 \quad X_{2}=X_{2}-N=-0,1092 \\
X_{3}=X_{3}-N=-0,0138 \quad X_{4}=X_{4}-N=0,0692 \\
G_{X}=\text { máximo }\left|X_{i}\right|=0,1092 \quad X_{1}=\frac{X_{1}}{G_{X}}=0,4158 \\
X_{2}=\frac{X_{2}}{G_{X}}=-1,0 \quad X_{3}=\frac{X_{3}}{G_{X}}=-0,1264 \quad X_{4}=\frac{X_{4}}{G_{X}}=0,6337
\end{gathered}
$$

El proceso continua hasta conseguir la convergencia. A continuación se resumen las ponderaciones "escores" en las diversas iteraciones, llegando a la solución óptima en la cuarta iteración. Observe también que $G_{X}$ y $G_{Y}$ convergen a los valores 0,2407 y 0,2613 respectivamente. 


\begin{tabular}{|c|c|c|c|c|c|}
\hline Columnas & 1 & 2 & 3 & 4 & 5 \\
\hline$X_{1}$ & 0 & 0,4158 & 1,0000 & 1,0000 & 1,0000 \\
\hline$X_{2}$ & 1 & $-1,0000$ & $-0,4718$ & 0,1842 & 0,1802 \\
\hline$X_{3}$ & 2 & $-0,1264$ & $-0,6348$ & $-0,6807$ & $-0,6808$ \\
\hline$X_{4}$ & 3 & 0,6337 & 0,8611 & 0,7696 & 0,7691 \\
\hline$G_{X}$ & 0,1092 & 0,1613 & 0,2487 & 0,2467 & 0,2407 \\
\hline Filas & 1 & 2 & 3 & 4 & 5 \\
\hline$Y_{1}$ & $-0,0454$ & $-0,3359$ & $-0,5550$ & $-0,7062$ & $-0,7055$ \\
\hline$Y_{2}$ & $-0,3510$ & 0,0964 & 0,4346 & 0,6683 & 0,6682 \\
\hline$Y_{3}$ & 1,0000 & 1,0000 & 1,0000 & 1,0000 & 1,0000 \\
$G_{Y}$ & 0,1675 & 0,2501 & 0,3368 & 0,2614 & 0,2613 \\
\hline
\end{tabular}

Nishisato (1988c) mostró que $\rho=\sqrt{G_{X} G_{Y}}=0,2508$, es la máxima correlación entre filas y columnas.

Según [7] la constante multiplicativa para ajustar la unidad de $Y_{1}, Y_{2}, Y_{3}$ es $C_{r}=1,3462$ y según [8], la constante multiplicativa para ajustar la unidad de $X_{1}, X_{2}$ y $X_{3}$ está dada por $C_{C}=1,4462$.

Las ponderaciones finales se obtienen multiplicando los pesos obtenidos en el último paso de las interacciones multiplicando por $C_{r}$ para $Y_{1}, Y_{2}, Y_{3}$ y por $C_{C}$ para $X_{1}, X_{2}$ y $X_{3}$. Estos nuevos pesos se denominan "pesos o ponderaciones normalizadas" $X_{i} C_{C}, Y_{i} C_{r}$, para los niveles de la especie drosophila (columnas): Huaylasi, Inca, Melanogaster y Novemaristata y para los niveles de las levaduras (filas): Saccharomyces, Candida utilis y Rhodotorula sp, respectivamente.

\begin{tabular}{|c|c|c|c|}
\hline \multicolumn{2}{|c|}{$\begin{array}{c}\text { Columnas } \\
\text { Ponderaciones normalizadas }\end{array}$} & \multicolumn{2}{c|}{ Ponderaciones normalizadas } \\
\hline$X_{1}$ & $1(1,4462)=1,4462$ & $Y_{1}$ & $-0,7055(1,3462)=-0,9497$ \\
\hline$X_{2}$ & $0,1802(1,4462)=0,2606$ & $Y_{2}$ & $0,6682(1,3462)=0,8995$ \\
\hline$X_{3}$ & $-0,6808(1,4462)=-0,9846$ & $Y_{3}$ & $1(1,3462)=1,3462$ \\
\hline$X_{4}$ & $0,7691(1,4462)=1,1123$ & \multicolumn{3}{|c}{} \\
\cline { 1 - 2 }
\end{tabular}

\subsection{Métodos de Análisis de Varianza}

Se identifican los elementos matriciales:

$$
\begin{gathered}
F=\left[\begin{array}{cccc}
17 & 51 & 162 & 63 \\
25 & 41 & 57 & 67 \\
12 & 6 & 24 & 38
\end{array}\right] \quad f_{C}=\left[\begin{array}{c}
54 \\
98 \\
243 \\
168
\end{array}\right] \quad f_{R}=\left[\begin{array}{c}
293 \\
190 \\
80
\end{array}\right] \quad \overrightarrow{1}=\left[\begin{array}{l}
1 \\
1 \\
1 \\
1 \\
1
\end{array}\right] \\
D_{C}=\left[\begin{array}{cccc}
54 & 0 & 0 & 0 \\
0 & 98 & 0 & 0 \\
0 & 0 & 243 & 0 \\
0 & 0 & 0 & 168
\end{array}\right] \quad D_{R}=\left[\begin{array}{ccc}
293 & 0 & 0 \\
0 & 190 & 0 \\
0 & 0 & 80
\end{array}\right] \quad f_{t}=563=n
\end{gathered}
$$


donde las ponderaciones óptimas que se buscan son $\vec{X}=\left[\begin{array}{c}X_{1} \\ X_{2} \\ X_{3} \\ X_{4}\end{array}\right]$ para las drosophilas, $\vec{Y}=\left[\begin{array}{c}Y_{1} \\ Y_{2} \\ Y_{3}\end{array}\right]$ para las levaduras.

Mediante el software Matlab se obtiene:

$$
\frac{f_{c} f^{\prime}: c}{f_{t}}=\left[\begin{array}{cccc}
5,1794 & 9,3996 & 23,3073 & 16,1137 \\
9,3996 & 17,0586 & 42,2984 & 29,2433 \\
23,3073 & 42,2984 & 104,8828 & 72,5115 \\
16,1137 & 29,2433 & 72,5115 & 50,1314
\end{array}\right]
$$

$$
G_{2}=\operatorname{trans} F * i n v D r * F=? ? ? ? ? ? ? ? ? ? ? ? ? ? ? ? ? ? ? ? ? ? ? ? ? ? ? ? ? ? ? ? ? ? ? ? ? ? ? ? ? ? ? ? ? ? ? ? ? ? ? ?
$$

\section{Conclusiones}

Fue posible la cuantificación de las categorías drosófilas y levaduras mediante las metodologías Método del Promedio Recíproco y Análisis de Varianza, pertenecientes a los denominados métodos descriptivos multivariantes no lineales, MUNDA. Sin embargo, dichas metodologías producen cuantificaciones diferentes para las categorías de las drosófilas y levaduras.

\section{Agradecimiento}

Al Consejo Superior de Investigaciones del Vicerrectorado de Investigación de laUNMSM, por el soporte financiero otorgado el año 2008 para la ejecución del proyecto de investigación cuyo resultado es la presente publicación. 


\section{REFERENCIAS BIBLIOGRÁFICAS}

[1] Belo,M.; LAVACA,P.(1982). Asociaçao entre drosophila e leveduras. Atraçao e produtividade. Naturalia. N0 7, 35- 45.

[2] Bishop,Y; Fiemberg, S. y Holland,P. (1975). Discrete multivariate analysis: Theory and Practice. The MIT Press, Cambridge, Massachusetts, USA.

[3] Cordeiro,A. (1990). Análise de dependencia. Tese de Livre Docencia. Universidade Estadual de Sao Paulo. Estado de Sao Paulo. Brasil.

[4] Gómez D. y et. al. (2006). Estudio de asociación entre drosophila y levaduras usando análisis de dependencia. PESQUIMAT. Revista de Investigación de la Facultad de Ciencias Matemáticas. UNMSM. Vol IX, N0 1, pág. 63-72.

[5] Greenacre, M. \& Blasius,J. (1994 ). Múltiple Correspondence Analysis and Related Methods. Boca Raton:Chapmanand Hall/CRC. New York.

[6] Grenacre M. (1984). Theory and applications of correspondence analysis. London: Academic Press.

[7] Greenacre, M. \& Blasius, J.(2006). Múltiple Correspondence Analysis in the Social Sciences. Academic Press. London.

[8] Grizzle,J.; Starmer, C. e Koch,G. (1969). Analysis of categorical data by linear models. Biometrics, 25, 489-504.

[9] Lebart,L.; Morineu, A. ; Fenelón, J. (1984). Multivariate descriptive statistical analysis. New York : John Wiley \& Sons.

[10] Nishisato, S. (2007). Multidimensional nonlinear descriptive análisis. Boca Raton: Chapman \& Hall/CRC. New York.

[11] Seber, G.(1984). Multivariate observations. John Willey, New York.

[12] Vásquez, J. (2002). Genética en poblaciones de Drosophila Fallén (Diptera-Hexapoda). Libro de artículos y resúmenes del I Congreso Peruano de Genética Animal: 62-64. Sociedad Peruana de Genética. 